\title{
Chlorophyll Mediated Photodynamic Inactivation of Blue Laser on Streptococcus Mutans
}

\author{
Suryani Dyah Astuti ${ }^{1, a)}$, A. Zaidan ${ }^{1}$, Ernie Maduratna Setiawati², \\ Suhariningsih ${ }^{1}$ \\ ${ }^{1}$ Department of Physics, Faculty of Science and Technology, Universitas Airlangga \\ ${ }^{2}$ Department of Periodontics, Faculty of Dentistry, Universitas Airlangga \\ ${ }^{a)}$ Corresponding author: suryanidyah@unair.ac.id
}

\begin{abstract}
Photodynamic inactivation is an inactivation method in microbial pathogens that utilize light and photosensitizer. This study was conducted to investigate photodynamic inactivation effects of low intensity laser exposure with various dose energy on Streptococcus mutans bacteria. The photodynamic inactivation was achieved with the addition of chlorophyll as photosensitizers. To determine the survival percentage of Streptococcus mutans bacteria after laser exposure, the total plate count method was used. For this study, the wavelength of the laser is $405 \mathrm{~nm}$ and variables of energy doses are 1.44, 2.87, 4.31, 5.74, 7.18, and 8.61 in $\mathrm{J} / \mathrm{cm}^{2}$. The results show that exposure to laser with energy dose of $7.18 \mathrm{~J} / \mathrm{cm}^{2}$ has the best photodynamic inactivation with a decrease of $78 \%$ in Streptococcus
\end{abstract}

Keywords: photodynamic inactivation, chlorophyll, photosensitizer, streptococcus mutans

\section{Introduction}

Dental caries is one of the most common infectious disease in the world. It can be caused by many factors, but bad life habits and infection of Streptococcus mutans are the most important factors $[1,2]$. Streptococcus mutans is a Gram-positive, facultative anaerobic bacterium. Because it is anaerobic, it is commonly found in the human oral cavity and is a significant contributor to tooth decay [3]. In the dental caries process, the bacterium metabolizes different kinds of carbohydrates, such as glucose and sucrose, creating an acidic environment in the mouth as a result of this process. Also, it enhances biofilm formation with the early colonizing bacteria to induce dental caries. This acidic environment in the mouth is what causes the tooth decay.

Therefore, control of the bacterial biofilm on teeth is very important for maintenance because it will cause various symptoms that affect our daily lives. As the bacteria develop in our mouth, they can cause tooth decay, multiple infections, and psychological problems such as low self-esteem which results in poor social interactions. For a long time, antibiotics such as penicillin are used to treat dental caries, but a shift from narrows spectrum antibiotic to broad-spectrum aminopenicillins which include amoxicillin causes increase in bacterial resistance to the former antibiotics [4]. Due to the increasing problem of antibiotics resistance, it is necessary to other alternative methods that are more effective and safe in maintaining oral health and prevent dental caries as well as eco-friendly, one of which is the photodynamic method.

In nature some of bacteria containing porphyrin compounds that are sensitive to light. In 2010, Papageorgiou et. al. show that exposure of light with a specific wavelength spectrum and dose of energy can lead to Photodynamic Inactivation (PDI) of bacteria [5]. The PDI mechanism involving photosensitizer light absorption process, i.e. light absorption process by bacterial porphyrin which further leads to the occurrence of advanced chemical reactions that produce a variety of reactive oxygen species [6]. PDI can disturb the activity of cell metabolism due to damage of cytoplasmic membrane which is induced by reactive oxygen in lipids and proteins resulting in lysis of the cells or

5th International Conference and Workshop on Basic and Applied Sciences (ICOWOBAS 2015) AIP Conf. Proc. 1718, 120001-1-120001-8; doi: 10.1063/1.4943353 (C) 2016 AIP Publishing LLC 978-0-7354-1364-1/\$30.00 